\title{
Nanoscale
}

RSCPublishing

Cite this: Nanoscale, 2013, 5, 8718

\section{Nanomagnet-based removal of lead and digoxin from living rats}

\author{
Inge K. Herrmann, $\dagger^{* a b}$ Andrea Schlegel, $\uparrow^{\mathrm{c}}$ Rolf Graf, ${ }^{\mathrm{c}}$ Christoph M. Schumacher, ${ }^{d}$ \\ Nico Senn, ${ }^{e}$ Melanie Hasler, ${ }^{\text {ab }}$ Sabrina Gschwind, ${ }^{f}$ Ann-Marie Hirt, ${ }^{e}$ Detlef Günther, ${ }^{\dagger}$ \\ Pierre-Alain Clavien, ${ }^{c}$ Wendelin J. Stark ${ }^{d}$ and Beatrice Beck-Schimmert:
}

\begin{abstract}
In a number of clinical conditions such as intoxication, bacteraemia or autoimmune diseases the removal of the disease-causing factor from blood would be the most direct cure. However, physicochemical characteristics of the target compounds limit the applicability of classical filtration and diffusion-based processes. In this work, we present a first in vivo magnetic blood purification rodent animal model and demonstrate its ability to rapidly clear toxins from blood circulation using two model toxins with stable plasma levels (lead $\left(\mathrm{Pb}^{2+}\right)$ and digoxin). Ultra-strong functionalized metal nanomagnets are employed to eliminate the toxin from whole blood in an extracorporeal circuit. In the present experimental demonstration over $40 \%$ of the toxin (i.e. lead or digoxin) was removed within the first 10 minutes and over $75 \%$ within 40 minutes. After capturing the target substance, a magnetic trap prevents the toxinloaded nanoparticles from entering the blood circulation. Elemental analysis and magnetic hysteresis measurements confirm full particle recovery by simple magnetic separation (residual particle concentration below $1 \mu \mathrm{g} \mathrm{mL}^{-1}$ (detection limit)). We demonstrate that magnetic separation-based blood purification offers rapid blood cleaning from noxious agents, germs or other deleterious materials with relevance to a number of clinical conditions. Based on this new approach, current blood purification technologies can be extended to efficiently remove disease-causing factors, e.g. overdosed drugs, bacteria or cancer cells without being limited by filter cut-offs or column surface saturation.
\end{abstract}

Received 13th May 2013

Accepted 8th July 2013

DOI: $10.1039 / \mathrm{c} 3 \mathrm{nr} 02468 \mathrm{~g}$

www.rsc.org/nanoscale potassium, urea and creatinine. With continuing identification of new disease-causing factors with higher molecular weight, i.e. proteins, antibodies or endotoxins, there is a great demand for new technological solutions to access these compounds. ${ }^{3}$

Recently, we and others have proposed a highly selective separation of target compounds from whole blood using functionalized magnetic nanoparticles. ${ }^{4-6}$ In the magnetic blood purification process, circulating target compounds bind to capturing agents immobilized on tiny magneto-responsive particles before the loaded nanoparticles are re-collected by magnetic separation. In contrast to classical purification processes using membranes or porous adsorbents, nanoparticles offer a high external surface area where capturing agents (e.g. antibodies) can be immobilized at a high density on the outermost surface layer, avoiding pore diffusion and providing excellent accessibility to the target. ${ }^{7,8}$ This geometry translates into highly efficient fluid purification, even for high molecular weight compounds with low diffusivity. In vitro

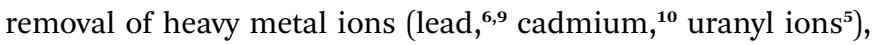
small molecule drugs (diazepam, ${ }^{4}$ digoxin $^{6}$ ), endotoxins, ${ }^{\mathbf{1 1}, \mathbf{1 2}}$ inflammatory mediators ${ }^{\mathbf{6 , 1 3}}$ or cells ${ }^{\mathbf{1 4 , 1 5}}$ from plasma or whole blood samples has been reported. However, (long-term) risks and possible side effects associated with the use of nanoparticles in vivo are not yet completely understood. ${ }^{16-19}$ Hence, 
an extracorporeal setting is preferred at this stage as it prevents nanoparticles from entering the body through the blood circuit. In an extracorporeal setting, nanoparticles are injected into an extracorporeal loop to promote the removal of target compounds. Prior to re-circulation of the blood into the body, a magnetic trap collects the particles, allowing for a continuous extracorporeal blood cleaning process. ${ }^{13}$

Although the number of in vitro studies investigating the removal of compounds from whole blood by magnetic separation has increased rapidly in the last five years, ${ }^{20}$ the concept has not yet been translated into an in vivo model. The implementation into a feasible in vivo process has most likely been constrained by the use of weakly magnetic particles hampering recovery, and the limited blood circulation time of intravascularly applied nanoparticles. ${ }^{21}$ In addition, blood coagulation issues and the limited blood volume of rodents may have prevented rapid advances in the field. ${ }^{22}$

Here, we present an in vivo magnetic blood purification rodent animal model and demonstrate its ability to rapidly clear toxins from blood circulation. Based on this new technology, new therapeutic targets may be accessed by blood purification.

\section{Results and discussion}

In this work, for the first time we show the successful implementation of a magnetic nanoparticle-based blood purification technology into a rodent animal model. As both the surface loading (the number of functional groups per surface unit) and recovery of magnetic particles are key determinants critical to the process efficiency, ultra-strong magnetic nanoparticles with non-oxidic iron cores are used as magnetic carriers.

\section{Synthesis and properties of functionalized nanomagnets}

Carbon-encapsulated platinum-spiked iron carbide $\left(\mathrm{C} / \mathrm{Fe}_{3} \mathrm{C}\right)$ nanomagnets were synthesized using a flame spray synthesis process. ${ }^{23}$ Addition of acetylene $\left(\mathrm{C}_{2} \mathrm{H}_{2}\right)$ to the flame enabled in situ carbon-coating of the nanoparticles thus preventing oxidation of the iron-based core and corresponding loss of magnetism. Synthesized nanomagnets are highly magnetic with saturation magnetizations of $\sim 123 \mathrm{emu}^{-1}$. Platinum is used as a tracer to facilitate particle detection by elemental analysis even in complex and iron-rich matrices. The outermost carbon layer surrounding the highly magnetic core was covalently functionalized with target-specific capturing moieties. In this first experimental demonstration, heavy metal ions (lead, $\mathrm{Pb}^{2+}$ ) and digoxin (small molecule drug) served as model compounds having stable plasma levels and commercially available capturing moieties (iminodiacetic acid-based chelator and digoxin antibody FAB (digiFAB)) (Fig. 1). Nanomagnets carrying target-specific capturing agents were dispersed in saline, a physiological carrier fluid to enable continuous injection into the circulating blood.

\section{Extracorporeal magnetic separation-based blood purification in rats}

Male Wistar rats with an estimated total blood volume of $25 \mathrm{~mL}$ were intoxicated by intravenous administration of the target

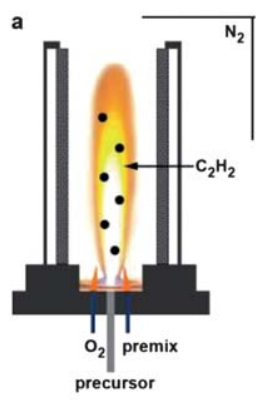

d

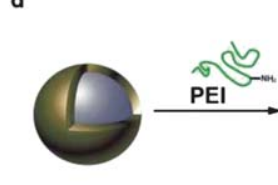

$$
\mathrm{C} / \mathrm{Fe}_{3} \mathrm{C}
$$

e

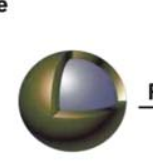

$\mathrm{C} / \mathrm{Fe}_{3} \mathrm{C}$

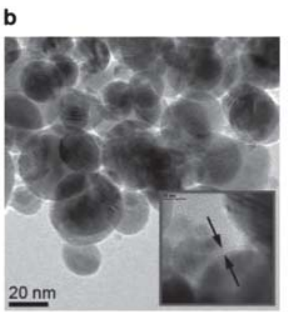

c
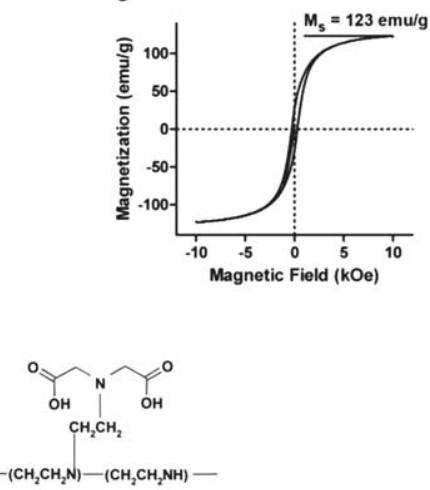

-PEI-IDA

Pb-specific nanomagnets

Digoxin-specific nanomagnets

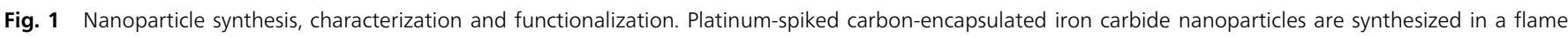

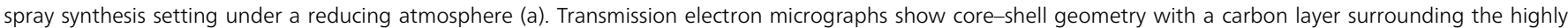

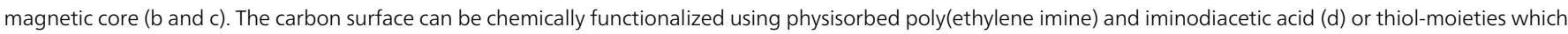
can be crosslinked with antibody fragments using a poly(ethylene glycol)-based crosslinker (e). 
toxin (lead $\left(0.16 \mathrm{mg} \mathrm{kg}^{-1}\right)$ or digoxin $\left.\left(0.01 \mathrm{mg} \mathrm{kg}^{-1}\right)\right)$. The left carotid artery was catheterized and connected through extracorporeal tubing to the left external jugular vein (Fig. 2a). The tubing and catheters were previously flushed with heparin. Blood was pumped through the extracorporeal circuit with a total extracorporeal volume of $2.8 \mathrm{~mL}$ at a flow rate of $1.5 \mathrm{~mL}$ $\min ^{-1}$ and functionalized magnetic nanoparticles were continuously injected into the circulating blood through a silicone injection port at a flow rate of $0.75 \mathrm{mg} \mathrm{min}^{-1}$ to reach a final concentration of $0.5 \mathrm{mg}$ nanoparticles per $\mathrm{mL}$ blood, i.e. about $30 \mathrm{mg}$ in total for one experiment. Before the blood entered the external jugular vein, the nanomagnets were collected and separated from the re-circulating blood using a magnetic separation unit (gradient field induced by a permanent magnet). Animals undergoing the same procedure without injection of functionalized nanomagnets but of pure saline instead served as controls to account for variations in plasma concentration due to dilution (extra volume added by nanomagnet injection) and tissue accumulation/metabolism/excretion. During the experiment, blood samples were sequentially collected every ten minutes and analyzed for remaining toxin concentrations by well-established assays used in clinical diagnostics. $^{24,25}$

\section{Toxin concentration measurements}

Lead ion plasma levels were found to be significantly decreased in animals undergoing magnetic separation based blood purification using target-specific nanomagnets compared to saline treated control animals (lead: $p<0.01, N=5$ ) (Fig. 2b). An additional control experiment using non-functionalized nanomagnets (no binding sites for lead ions) demonstrated a complete lack of purification. Similarly, digoxin could be rapidly removed from blood using digoxin-specific nanomagnets $(p=0.0286, N=5$ ) (Fig. 2c). For both model compounds, over $40 \%$ of the toxin was removed within the first 10 minutes $(p<0.001)$ and over $75 \%$ within 40 minutes. Toxin plasma levels in control animals remained relatively stable over a time period of 40 minutes, with a decrease by $10 \%$ for lead and $32 \%$ for digoxin, respectively.

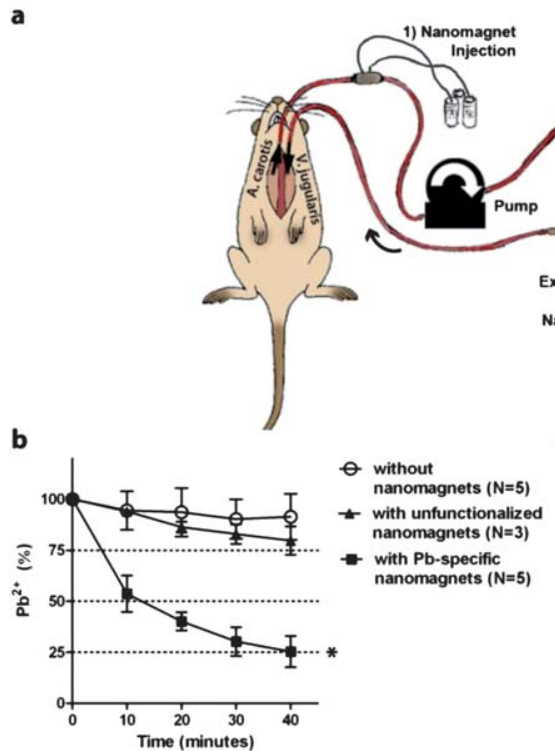

c
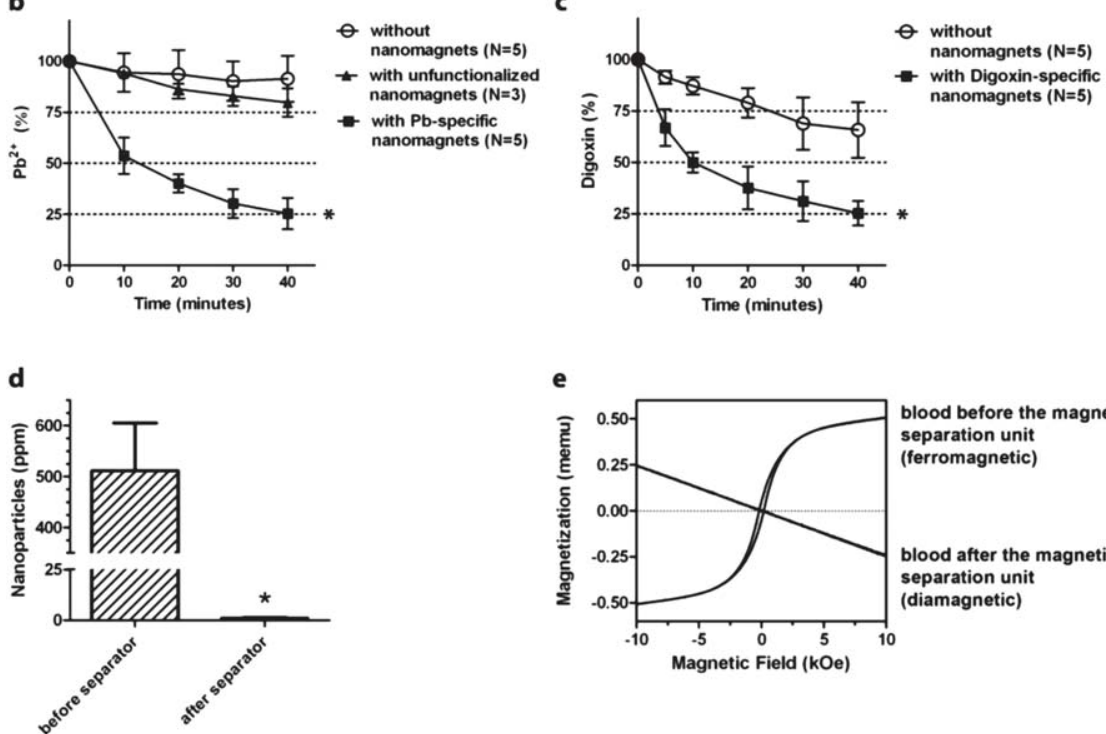

e

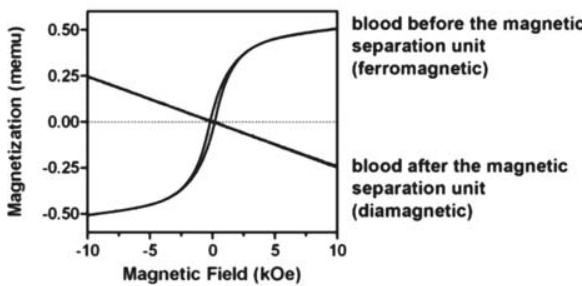

Fig. 2 In vivo magnetic separation based blood purification (a). Rats were catheterized placing catheters into the left carotid artery and external jugular vein gaining access to the blood circuit. The catheters were connected to the extracorporeal purification assembly consisting of blood transfusion tubing, a silicone port for nanomagnet injection and a magnetic separation unit. In magnetic blood purification experiments using target-specific nanomagnets, concentrations of lead (b) and digoxin (c) decreased rapidly compared to controls (lead: $p<0.01$; digoxin: $p=0.0286$ ). Unfunctionalized nanomagnets (no binding sites) were ineffective. Platinum content in blood samples after chemical digestion of the platinum-spiked nanoparticles was below the detection limit (d). Magnetic hysteresis measurements confirm the high recovery of nanomagnets by the magnetic separation unit: before the magnetic separator, the blood exhibits ferromagnetic properties while after separation it is diamagnetic (e). 


\section{Magnetic separation of nanomagnets before blood recirculation}

To verify how efficiently nanomagnets were eliminated from the blood stream, blood was collected after the magnetic separation unit and analyzed for residual particle concentration by measuring the platinum content after chemical digestion by inductively coupled plasma-mass spectrometry (ICP-MS). The platinum concentration in the blood sample after the magnetic separation unit was found below limits of detection (detection limit of platinum in whole blood: $\leq 1 \mathrm{ng}$ platinum per $\mathrm{mL}$ corresponding to $\leq 1 \mu \mathrm{g}$ nanoparticles per $\mathrm{mL}$ blood) (Fig. 2d). Analysis of magnetic properties of whole blood samples, i.e. hysteresis measurements, showed that blood before the magnetic separation unit exhibited ferromagnetic properties while after the separation unit, blood samples were diamagnetic (Fig. 2e). This discrete change in properties is in good agreement with elemental analysis and confirms the high separation efficiency using a magnetic separator with simple geometry. Thus, magnetic measurements, in contrast to elemental analysis, enable identification of the iron phase of interest (i.e. intact nanomagnets) without the need for chemical digestion, and differentiation between nanomagnets and dissolved iron.

\section{Safety issues - what happens if the magnetic separation fails?}

To address safety issues concerning incomplete removal of nanomagnets, we analyzed tissue from animals after treatment
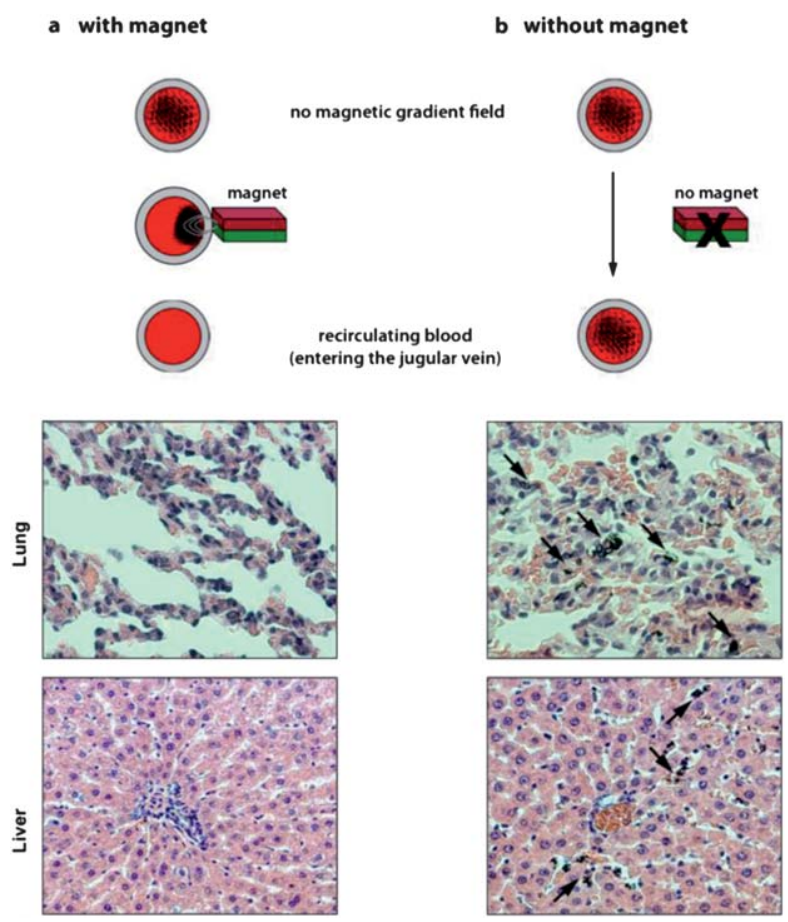

Fig. 3 Histological examination of lung and liver from rats undergoing extracorporeal blood purification with or without magnetic separation of the nanomagnets before recirculation (H\&E staining). If nanomagnets are separated prior to re-entry into the body, histological images show no accumulation of nanomagnets in tissue (a). If nanomagnets are not separated before re-entry into the body (no magnet), nanomagnets are detected in both lung and liver tissues (black arrows) (b). with nanomagnets which were removed using the separator unit. Organs, particularly the liver with its phagocytic activity, were collected and analyzed for accumulated nanomagnets originating from potentially incomplete nanoparticle separation by the magnetic separation unit. Histological analysis of liver tissue visually confirmed the absence of nanoparticles (Fig. 3). This is in good agreement with a recent study showing that metal nanomagnets enable near quantitative collection efficiency. ${ }^{26}$ In contrast, when the worst case scenario was explored by omission of the separation unit for 15 minutes, analysis of lung and liver tissue samples showed significant accumulation of particles. Hence, extracorporeal application remains the preferred setting in terms of accessibility, efficiency and safety.

\section{Conclusions}

In summary, we present an easily applicable implementation of a nanomagnet-based blood purification process in a first in vivo detoxification model. Blood can be successfully purified in an extracorporeal blood purification circuit using functionalized nanomagnets as capturing moieties. Remarkable separation efficiencies are achieved even with a simple magnetic separation unit using a single permanent magnet. Hence, in vivo magnetic separation-based blood purification is a potent new method to selectively remove noxious compounds from blood not limited by size-selection criteria. This new technological solution provides the basis for new therapeutic targets and may be extended to selectively isolate disease causing factors such as overdosed drugs, proteins, endotoxins, bacteria (e.g. in sepsis) or cancer cells in a clinical setting.

The extracorporeal application of nanoparticles can be considered as a first step towards a safe implementation of nanomagnet-based treatments provided that safety barriers are implemented. Iron-based particle detection in complex and iron-rich matrices presents a major challenge even for the best available elemental analysis devices. Magnetic hysteresis measurements offer a valuable alternative complementing elemental analysis without requiring nanoparticle digestion. With regard to future clinical applications, the magnetic separation efficacy is pivotal: the safe implementation of this young technology relies on the minimal risk policy by limiting direct exposure to nanomagnets and a detailed risk evaluation. This includes assessment of acute and long-term adverse effects and more advanced particle detection methods., ${ }^{\mathbf{1 , 2 6 - 2 8}}$

\section{Experimental}

Synthesis and characterization of carbon encapsulated

Carbon encapsulated platinum-spiked iron carbide nanomagnets were synthesized using flame spray synthesis under a reducing atmosphere (detailed synthesis procedure can be found elsewhere) ${ }^{29}$ Briefly, iron and platinum precursors were synthesized by dissolving iron nitrate and platinum acetylacetonate in 2-ethylhexanoic acid, respectively. The precursors were mixed to obtain a mass content of $0.1 \%$ platinum, diluted with 
tetrahydrofuran (THF, Fluka, technical grade, $2: 1$ ) and filtered. Carbon encapsulated platinum-spiked iron carbide nanoparticles were synthesized in a flame spray synthesis reactor under a nitrogen atmosphere. The precursor was fed into the spray nozzle at a flow rate of $6 \mathrm{~mL} \mathrm{~min}^{-1}$. Acetylene (Pangas) was added at a flow rate of $5 \mathrm{~L} \mathrm{~min}^{-1}$ to obtain in situ coating of the nanoparticles. As-prepared nanoparticles were thoroughly washed in $24 \mathrm{vol} \%$ hydrochloric acid ( $\mathrm{HCl}$, Fluka, puriss) to dissolve incompletely coated nanoparticles. The platinum content was measured using inductively coupled plasma mass spectrometry (ICP-MS, Element2, Thermo-Finnigan, Bremen, Germany). Magnetic hysteresis measurements were performed on a vibrating sample magnetometer (VSM, Princeton Measurements Corporation, model 3900) using a $100 \mathrm{~ms}$ averaging time.

\section{Chemical functionalization}

For lead-ion selective functionalization, nanomagnets were coated with poly(ethylene imine) (PEI)-iminodiacetic acid (IDA). Before branched PEI (10 kDa, Polysciences Inc., 99\%) was physisorbed on the carbon shell encapsulating the nanoparticles, the particles were washed in $\mathrm{H}_{2} \mathrm{O}$ (Millipore) and DMF (Fluka, puriss), respectively. PEI was dissolved in dimethylformamide (DMF), added to nanoparticles pre-dispersed in DMF and stirred overnight. Then, the nanoparticles were washed in DMF, water and acetone (Fluka, puriss) three times each and dried in a vacuum. Subsequently, nanoparticles (100 $\mathrm{mg})$ were dispersed in DMF $(100 \mathrm{~mL})$, and potassium hydroxide (0.2 g, Sigma Aldrich) and bromoacetic acid (0.1 g, Sigma Aldrich) were added. The mixture was stirred at 90-110 ${ }^{\circ} \mathrm{C}$ and $5 \mathrm{~mL}$ of $\mathrm{H}_{2} \mathrm{O}$ were added. Nanoparticles were separated from the dispersion, washed and characterized using infrared spectroscopy (identification of functional groups, Fourier transform IR (FTIR)) using a Tensor 27 Spectrometer (Bruker Optics, equipped with a diffuse reflectance accessory, DiffusIR, Pike technologies), and elemental analysis (quantitative analysis of carbon and nitrogen content, LECO, CHN-900). For antidigoxin functionalization, nanomagnets were functionalized with thiol moieties, and then crosslinked with digiFAB using a heterobifunctional crosslinker (MAL-dPEG 24 -NHS, Quantabiodesign). ${ }^{13}$

\section{Implementation of the extracorporeal blood circuit in Wistar rats}

An extracorporeal circuit was assembled using tygon tubing with an ID of $3 \mathrm{~mm}$ and a length of $40 \mathrm{~cm}$. A silicone injection port for nanoparticle-injection was connected. Both tube endings were connected to fine bore polyethylene catheters (0.58 mm ID, $0.96 \mathrm{~mm}$ OD) using 23G needles. The extracorporeal circuit was flushed with saline containing heparin $(500 \mathrm{U}$ $\mathrm{mL}^{-1}$ ) prior to operation of the animals.

Animal experiments were performed in accordance with Swiss Federal Animal Regulations and approved by the Veterinary Office of Zurich, Switzerland (no. 157/2011). Male Wistar rats weighing 350-500 g (Charles River, Sulzfeld, Germany) were used. The rats were housed in standard cages at $22 \pm 1{ }^{\circ} \mathrm{C}$ under a
12/12 h light-dark regimen. Food and water were supplied ad libitum. Rats were weighed and anesthetized with 3\% isoflurane (Attane, Minrad Inc., Buffalo, NY, USA). The ventral surface of the neck area, off midline and just above the external jugular vein, was shaved, cleaned, and de-germed. A small incision was made for access and cannulation of the left carotid artery and the external jugular vein. Both artery and vein were surgically exposed and two sutures (6-0 silk), proximal and distal, were placed around the vessels. First, the distal suture of the carotid artery was retracted followed by a small incision into the ventral vessel wall, where the catheter was inserted and stabilized using the second silk suture. Blood was pumped into the extracorporeal circuit (pump from H. J. Guldener, Zurich, ZU/83W, 12082). The opponent ending of the tubing system was inserted into the external jugular vein and blood was pumped through the circuit

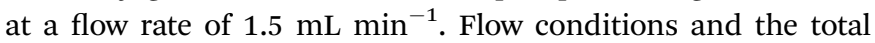
extracorporeal volume were adapted from Adachi et al. ${ }^{\mathbf{3 0 , 3 1}}$ Experimental groups include at least five animals each $(N=5)$.

\section{Toxin concentration measurements}

The first blood sample was collected 10 minutes after intravenous administration of the toxin in the jugular vein before magnetic blood purification was started. Subsequently, blood samples of $0.5 \mathrm{~mL}$ were collected every 10 minutes. Blood samples were analyzed for remnant toxin concentrations using Atomic Absorption Spectrometry (AAS) for lead ions ${ }^{24}$ and a kinetic interaction of microparticles in solution assay (KIMS, Roche Diagnostics) for digoxin. ${ }^{25}$

\section{Nanoparticle concentration measurements}

Nanoparticle residues were determined using two complementary methods, elemental analysis and magnetic hysteresis measurements. For ICP-MS measurements (Element2, Thermo Fisher Scientific, Bremen), biological samples (blood and tissues) were digested using hydrochloric acid (conc.), nitric acid (conc.), and hydrogen peroxide $(30 \%)$ at $200{ }^{\circ} \mathrm{C}$ and $40 \mathrm{bar}$ ensuring total dissolution of all particles and the biological matrix. Platinum contents were then determined using ICP-MS. Magnetic hysteresis measurements of freeze-dried blood samples (CHRIST Alpha 1-4 freeze dryer, $<0{ }^{\circ} \mathrm{C} /<6.1 \mathrm{mbar}$ ) were performed on a vibrating sample magnetometer (VSM, Princeton Measurements Corporation, model 3900) using a $100 \mathrm{~ms}$ averaging time.

\section{Histology}

In all groups, following blood purification, organs were procured and lung and liver tissue were analyzed using standard formalin fixation and H\&E staining methods at magnifications of 10x, 20x and 40x.

\section{Statistics}

Plasma concentrations of toxins and nanoparticle concentrations are plotted as means \pm standard deviation. Plasma concentrations were compared based on area under the curve (AUC) values using Mann-Whitney-U tests in SPSS. 


\section{Acknowledgements}

We thank Mrs Lea Schütz-Cohen for helpful support. We kindly acknowledge financial support from the Swiss National Science Foundation (NFP-64, grant no. 131268), the Forschungskredit of the University of Zurich (grant no. 54345802, I.K.H.) the Hartmann-Müller Foundation (grant no. 34345825, I.K.H.) and the Herzog-Egli Foundation (grant no. 34345824, I.K.H.).

\section{Notes and references}

1 W. J. Stark, Angew. Chem., Int. Ed., 2011, 50, 1242.

2 J. Himmelfarb and T. A. Ikizler, N. Engl. J. Med., 2010, 363, 1833.

3 K. von Appen, C. Weber, U. Losert, H. Schima, H.-J. Gurland and D. Falkenhagen, Artif. Organs, 1996, 20, 420.

4 K. Cai, J. Li, Z. Luo, Y. Hu, Y. Hou and X. Ding, Chem. Commun., 2011, 47, 7719.

5 L. Wang, Z. Yang, J. Gao, K. Xu, H. Gu, B. Zhang, X. Zhang and B. Xu, J. Am. Chem. Soc., 2006, 128, 13358.

6 I. K. Herrmann, M. Urner, F. M. Koehler, M. Hasler, B. RothZ'graggen, R. N. Grass, U. Ziegler, B. Beck-Schimmer and W. J. Stark, Small, 2010, 6, 1388.

7 J. Klein, Proc. Natl. Acad. Sci. U. S. A., 2007, 104, 2029.

8 Y. Pan, X. Du, F. Zhao and B. Xu, Chem. Soc. Rev., 2012, 41, 2912.

9 H. Y. Lee, D. R. Bae, J. C. Park, H. Song, W. S. Han and J. H. Jung, Angew. Chem., Int. Ed., 2009, 48, 1239.

10 J. Jin, F. Yang, F. W. Zhang, W. Q. Hu, S. B. Sun and J. T. Ma, Nanoscale, 2012, 4, 733.

11 L. Bromberg, E. P. Chang, C. Alvarez-Lorenzo, B. Magarinos, A. Concheiro and T. A. Hatton, Langmuir, 2010, 26, 8829.

12 I. K. Herrmann, M. Urner, S. Graf, C. M. Schumacher, B. Roth-Z'graggen, M. Hasler, W. J. Stark and B. BeckSchimmer, Adv. Healthcare Mater., 2013, 2, 829.

13 I. K. Herrmann, R. E. Bernabei, M. Urner, R. N. Grass, B. Beck-Schimmer and W. J. Stark, Nephrol., Dial., Transplant., 2011, 26, 2948.

14 H. Xu, Z. P. Aguilar, L. Yang, M. Kuang, H. Duan, Y. Xiong, H. Wei and A. Wang, Biomaterials, 2011, 32, 9758.
15 M. Y. Sha, H. Xu, M. J. Natan and R. Cromer, J. Am. Chem. Soc., 2008, 130, 17214.

16 A. Nel, T. Xia, L. Mädler and N. Li, Science, 2006, 311, 622.

17 P. Hoet, I. Bruske-Hohlfeld and O. Salata, J. Nanobiotechnol., 2004, 2, 12.

18 A. Stampfl, M. Maier, R. Radykewicz, P. Reitmeir, M. Göttlicher and R. Niessner, ACS Nano, 2011, 5, 53455353.

19 G. Oberdorster, E. Oberdorster and J. Oberdorster, Environ. Health Perspect., 2005, 113, 823.

20 L. M. Graham, T. M. Nguyen and S. B. Lee, Nanomedicine, 2011, 6, 921.

21 C. C. Berry and A. S. G. Curtis, J. Phys. D: Appl. Phys., 2003, 36, R198.

22 A. Radomski, P. Jurasz, D. Alonso-Escolano, M. Drews, M. Morandi, T. Malinski and M. W. Radomski, Br. J. Pharmacol., 2005, 146, 882.

23 R. N. Grass, E. K. Athanassiou and W. J. Stark, Angew. Chem., Int. Ed., 2007, 46, 4909.

24 B. E. Jacobson, G. Lockitch and G. Quigley, Clin. Chem., 1991, 37, 515.

25 S. M. Palmer, R. A. Kaufman, S. J. Salamone, J. BlakeCourtney, W. Bette, H. P. Wahl and F. Furrer, Clin. Chem., 1995, 41, 1751.

26 C. M. Schumacher, I. K. Herrmann, S. B. Bubenhofer, S. Gschwind, A.-M. Hirt, B. Beck-Schimmer, D. Günther and W. J. Stark, Adv. Funct. Mater., 2013, DOI: 10.1002/ adfm. 201300696.

27 A. Nel, D. Grainger, P. Alvarez, S. Badesha, V. Castranova, M. Ferrari, H. Godwin, P. Grodzinski, J. Morris, N. Savage, N. Scott and M. Wiesner, in Nanotechnology Research Directions for Societal Needs in 2020, Springer Netherlands, 2011, vol. 1, pp. 159-220.

28 B. Pelaz, G. Charron, C. Pfeiffer, Y. Zhao, J. M. de la Fuente, X.-J. Liang, W. J. Parak and P. del Pino, Small, 2013, 9, 1573.

29 I. K. Herrmann, R. N. Grass, D. Mazunin and W. J. Stark, Chem. Mater., 2009, 21, 3275.

30 T. Adachi, M. Mogi, M. Harada and K. Kojima, J. Chromatogr., Biomed. Appl., 1995, 668, 327.

31 T. Adachi, M. Mogi, M. Furuya and K. Kojima, Toxicol. Lett., 2000, 115, 63. 